\title{
In Defense of Unification (Comments on West and Koch's review of Causality)
}

\author{
Judea Pearl \\ University of California, Los Angeles \\ Computer Science Department \\ Los Angeles, CA, 90095-1596, USA \\ (310) 825-3243 / judea@cs.ucla.edu
}

A new review of my book Causality (Pearl, 2009) has appeared in the Journal of Structural Equation Modeling (SEM), authored by Stephen West and Tobias Koch (W-K) West and Koch (2014).

I find the main body of the review quite informative, and I thank the reviewers for taking the time to give SEM readers an accurate summary of each chapter, as well as a lucid description of the key ideas that tie the chapters together. However, when it comes to accepting the logical conclusions of the book, the reviewers seem reluctant, and tend to cling to traditions that lack the language, tools and unifying perspective to benefit from the chapters reviewed.

The reluctance culminates in the following paragraph: "We value Pearl's framework and his efforts to show that other frameworks can be translated into his approach. Nevertheless we believe that there is much to be gained by also considering the other major approaches to causal inference."

W-K seem to value my "efforts" toward unification, but not the unification itself, and we are not told whether they doubt the validity of the unification, or whether they doubt its merits. Or do they accept the merits and still see "much to be gained" by pre-unification traditions? If so, what is it that can be gained by those traditions and why can't these gains be achieved within the unified framework presented in Causality?

In their explanation of why they do not embrace the unification whole-heartedly, they seem to be speaking about another book entirely, not the one I have written. In their words: "[In Pearl's framework] there are relatively few unknown causal influences in the system, the units tend to be homogeneous, causal effects are assumed to be stable across settings and time, and levels of treatments can be set to specified values as reflected in the $d o(x)$ operator..."

This description would come as a total surprise to readers of Causality, because none of the listed shortcomings apply to the methods described in the book, as I will soon elaborate, and as would be attested by anyone who tries to solve concrete problems using several alternative methods. To the best of my knowledge, chapter 7 of Causality (especially pp. 231234 ) is still the only text available in which concrete problems are solved side by side in both 
the structural and potential outcome approaches. I invite readers to examine, for example, the smoking-tar-cancer story on page 83 of Causality and determine, using "other major approaches" whether the effect of smoking on cancer can be estimated from the assumed model. It is a simple example, only a few lines of text, but truly instructive; Heckman and Pinto have labored over 20 full pages trying to fit and solve it within "traditional econometrics" (2014).

Thus, can the statement "much to be gained by other major approaches" be defended without trying out those approaches on concrete examples?

I assume W-K reached their first conclusion (that the structural approach assumes few unknown causal influences) by counting variables in the examples illustrated in Causality and comparing them to the hundreds of "unknown causal influences" that haunt the complex real-life problems that they and other researchers are undoubtedly "very concerned about." But if one accepts the logical equivalence of the structural and potential outcome approaches, then surely the same assumptions that permit a potential outcome practitioner to ignore the many "unknown causal influences" in a messy practical problem can also be invoked in the structural approach, and achieve identical results. The only difference would be the transparency of the justification.

In fact, I am not aware of a system with many causal influences that can be analyzed through any other representation language except structural equations. Specifically, I have not seen ignorability assumptions ever justified in a system with more than 3-4 variables. (Skeptics are invited to try it on Fig. 7.5 on p. 232 of Causality.) What, then, is the utility of boasting about being "very concerned about" many "unknown causal influences" if one cannot handle even a toy problem with 3-4 causal influences? And what is the utility of comparing variable counts in textbook exercises versus unknown variables in ill-understood real life problems?

I am similarly puzzled by W-C's other findings. For example: "[In Pearl's framework] the units tend to be homogeneous." The opposite is in fact the case; units heterogeneity is assumed a priori in nonparametric models. See also Pearl (2012).

Or, "[In Pearl's framework] causal effects are assumed to be stable across settings and time." Not so. Causal effects are allowed to vary violently across settings (see page 113 and pp. 354-355) and, when dynamic treatments are concerned (as in chapter 4), they are allowed to vary across time as well.

Or, "levels of treatments can be set [externally] to specified values." Not so! Levels of treatments need not be set at all, as in the case of noncompliance (chapter 8) where the exposure $X=x$ may not result from direct intervention. The $d o(x)$ operator does not require one to imagine the physical manipulation of $X$ (as is required in the potential outcome approach). It is merely a notational device that distinguishes counterfactual conditionals from probabilistic conditionalization. I am yet to see a policy question that cannot be expressed using the $d o(x)$ operator or its unit-level companions - counterfactual conditionals.

$\mathrm{W}-\mathrm{K}$ present the following arguments in favor of Rubin's potential outcomes approach:

(i) "Rubin's potential outcomes model defines a causal effect as the difference between the response of a single unit given treatment or control at the same time and in the same context."

Since the structural definition of $Y_{x}(u)$ is logically equivalent to Rubin's $Y_{x}(u)$, why 
should not the former inherit all the merits of the latter? It indeed does. Chapter 9 and pp. 396-398 define and deal with unit-level counterfactuals, and show that, contrary to conventional wisdom, properties of unit-level counterfactuals can be inferred from population data. (See also Pearl $(2013,2014)$.)

(ii) "Rubin does not make homogeneity assumptions." Nor does the structural approach, as explained above.

(iii) "Rubin's perspective also offers procedures for addressing situations in which assumptions fail (e.g., treatment noncompliance)."

Treatment noncompliance is not a situation in which assumptions fail. Rather, it is a situation in which the assumptions are different than those made in controlled randomized trials and, not surprisingly, by being explicit about the new set of assumptions, the structural framework has produced some of the most ambitious conclusions that partially identified situations would permit. For example, chapter 8 of Causality derives universal bounds for $\mathrm{ACE}$ and ETT under noncompliance. It then supplements them with instrumental inequalities, as well as with vivid illustrations of how the Bayesian posterior of ACE varies with prior assumptions and with sample size. These developments were hardly shy of "addressing situations in which assumptions fail (e.g., treatment noncompliance)."

(iv) "Rubin often works in the environment of health research."

My latest readings of the health research literature assure me that epidemiologists and bio-statisticians have adopted DAGs as a preferred communication language (See e.g., (Glymour, 2006; Howards et al., 2012; Rothman et al., 2008; Wilcox, 2006). Social and behavioral scientists are not far behind (see, e.g., Lee (2012); Morgan (2013); Morgan and Winship (2007); Muthén (2014). I am surprised that W-C are not aware of this trend.

As another alternative approach to causal inference, W-K hail Campbell's perspective which I, confessedly, have not been able to use in my research. True, Campbell "offers researchers lists of threats to internal [and external] validity." These lists were probably very useful three decades ago, prior to the mathematization of causality. But I am curious to learn how they can be used today, when new tools are available, which permit us to detect and neutralize threats with mathematical precision. Of course, I would not rule out the possibility that Campbell's lists are still used by researchers who seek heuristic shortcuts to circumvent formal analysis. Such shortcuts benefit many sciences, and can be practiced in harmony with the formal approach, as long as we understand the boundaries of their applicability. (I am reminded of the laws of thermodynamics that were formulated and practiced prior to their derivation from the more fundamental principles of statistical mechanics, and are still used today by engineers and physicists). What we need therefore is a detailed analysis of how Campbell's heuristics follow from the structural theory of causation. and in what types of situations they provide good approximations to the latter. Such analysis would be illuminating, and I hope someone from Campbell's camp undertakes this task.

At the same time, we must acknowledge that it was graphical methods that produced solutions to the two problems that Campbell and Rubin set out to solve three or four decades ago: external validity, in the case of Campbell, and internal validity (or control of 
confounding) in the case of Rubin. The first is mathematized in (Pearl and Bareinboim, 2014) and the second in (Shpitser and Pearl, 2008). Threats may warn us of problems - they do not solve problems.

Finally, W-K's review ends with a recommendation that is both puzzling and revealing. It states: "We encourage SEM researchers following Pearl's framework to be skeptical, and to fiercely confront their preferred model with strong alternative models in the tradition of economics."

The puzzling part is that this recommendation is addressed to "followers of Pearl's framework" and not to those following the alternative perspectives of Rubin or Campbell. After all, Rubin too makes modeling assumptions (in W-K's words: "Rubin assumes strong ignorability..."), so why do we not hear an encouragement to be skeptical of these ignorability assumptions as well?

I know the answer, and I think W-K know it too - one cannot be skeptical of assumptions that one does not comprehend. Ignorability assumptions are cognitively formidable, hence they are not meant to be understood and to be judged for plausibility They are made "because they justify the use of available statistical methods and not because they are truly believed" (Joffe et al., 2010). By contrast, these same assumptions are shining loud and clear in the structural approach, and can therefore be submitted to skeptical scrutiny. Moreover, the structural representation explicates the testable implications of its modeling assumptions, while the potential outcome representation does not.

To summarize: If you are a follower of the structural approach, you should take W$\mathrm{K}$ recommendation with great pride. You were singled out to be skeptical of your model, because you are uniquely capable of explicating and interpreting your modelling assumptions and uniquely capable of testing their implications - your rivals can't.

I invite Stephen West and Tobias Koch to an open discussion on whether any other interpretation of their recommendation should be considered, given the unique capabilities of the methodology advanced in Causality

\section{Acknowledgment}

This research was supported in parts by grants from NIH \#1R01 LM009961-01, NSF \#IIS0914211 and \#IIS-1018922, and ONR \#N000-14-09-1-0665 and \#N00014-10-1-0933.

\section{References}

Glymour, M. M. (2006). Using causal diagrams to understand common problems in social epidemiology. In Methods in Social Epidemiology. John Wiley and Sons, San Francisco, CA, 393-428.

Heckman, J. J. and Pinto, R. (2014). Causal analysis after haavelmo. Econometric Theory Http://dx.doi.org/10.1017/S026646661400022X, Published online: 10 June 2014, FirstView Articles, pp. 1-37. 
Howards, P. P., Schisterman, E. F., Poole, C., Kaufman, J. S. and Weinberg, C. R. (2012). "toward a clearer definition of confounding" revisited with directed acyclic graphs. American Journal of Epidemiology 176 506-511.

Joffe, M. M., Yang, W. P. and Feldman, H. I. (2010). Selective ignorability assumptions in causal inference. The International Journal of Biostatistics 6. DOI: 10.2202/15574679.1199 .

LEe, J. (2012). Correlation and causation in the study of personality. European Journal of Personality 26 372-390.

Morgan, S. (2013). Handbook of Causal Analysis for Social Research. Springer, New York, NY.

Morgan, S. and Winship, C. (2007). Counterfactuals and Causal Inference: Methods and Principles for Social Research (Analytical Methods for Social Research). Cambridge University Press, New York, NY.

MuthÉN, B. (2014). Applications of causally defined direct and indirect effects in mediation analysis using SEM in Mplus. Tech. rep., Graduate School of Education and Information Studies, University of California, Los Angeles, CA. Forthcoming, Psychological Methods.

Pearl, J. (2009). Causality: Models, Reasoning, and Inference. 2nd ed. Cambridge University Press, New York.

Pearl, J. (2012). Detecting latent heterogeneity. Tech. Rep. R-406, $<$ http://ftp.cs.ucla.edu/pub/stat_ser/r406.pdf>, Department of Computer Science, University of California, Los Angeles, CA.

Pearl, J. (2013). The curse of free-will and paradox of inevitable regret. Journal of Causal Inference $1255-257$.

Pearl, J. (2014). Causes of effects, from philosophy to science. Tech. Rep. R-431, $<$ http://ftp.cs.ucla.edu/pub/stat_ser/r431.pdf >, Department of Computer Science, University of California, Los Angeles, CA. Short version forthcoming, Journal of Sociological Methods and Research.

Pearl, J. and Bareinboim, E. (2014). External validity: From docalculus to transportability across populations. Tech. Rep. R-400, $<$ http://ftp.cs.ucla.edu/pub/stat_ser/r400.pdf >, University of California Los Angeles, Computer Science Department, CA. Forthcoming Statistical Science.

Rothman, K., Greenland, S. and Lash, T. (2008). Modern Epidemiology. 3rd ed. Lippincott, Williams \& Wilkins, Philadelphia, PA.

ShPitser, I. and Pearl, J. (2008). Complete identification methods for the causal hierarchy. Journal of Machine Learning Research 9 1941-1979. $<$ http://ftp.cs.ucla.edu/pub/stat_ser/r336.pdf $>$. 
West, S. G. and Koch, T. (2014). Book review: Restoring causal analysis to structural equation modeling (review of causality, 2nd edition). Structural Equation Modeling: A Multidisciplinary Journal 21. Http://bayes.cs.ucla.edu/BOOK-2K/west-kochreview2014.pdf, DOI: 10.1080/10705511.2014.856700.

Wilcox, A. (2006). The perils of birth weight - a lesson from directed acyclic graphs. American Journal of Epidemiology $1641121-1123$. 